\title{
Condições de saúde e características do trabalho de enfermeiros de um hospital universitário*
}

\author{
Health conditions and Nurses' work characteristics at a university hospital
}

\section{Como citar este artigo:}

Pimenta CJL, Vicente MC, Ferreira GRS, Frazão MCLO, Costa TF, Costa KNFM. Health conditions and Nurses' work characteristics at a university hospital. Rev Rene. 2020;21:e43108. DOI: https://doi.org/10.15253/2175-6783.20202143108

\author{
(DCláudia Jeane Lopes Pimenta ${ }^{1}$ \\ (D) Mateus Carneiro Vicente ${ }^{1}$ \\ (D) Gerlania Rodrigues Salviano Ferreira ${ }^{1}$ \\ (1) Maria Cristina Lins de Oliveira Frazão ${ }^{1}$ \\ (1)Tatiana Ferreira da Costa $^{1}$ \\ DKátia Neyla de Freitas Macedo Costa $^{1}$
}

\begin{abstract}
RESUMO
Objetivo: analisar o perfil sociodemográfico, as condições de saúde e as características do trabalho de enfermeiros. Métodos: estudo transversal, realizado com 152 enfermeiros de um hospital universitário. Utilizou-se de instrumento estruturado, o qual foi analisado por estatística descritiva. Resultados: os enfermeiros consideraram a saúde como boa $(50,7 \%)$, realizavam atividade física $(53,3 \%)$, não fumavam $(92,8 \%)$, não consumiam álcool (56,6\%), apresentavam algum problema de saúde $(63,2 \%)$ e utilizam medicamentos diariamente $(54,6 \%)$. Observou-se maior quantitativo de profissionais do ambulatório (17,8\%) e clínica médica $(15,8 \%)$, que atuavam no hospital estudado por até cinco anos $(61,2 \%)$, celetistas $(74,3 \%)$, sem outro vínculo empregatício $(54,6 \%)$, do turno diurno $(56,6 \%)$, com escala de seis horas $(36,2 \%)$, que referiram ausência do trabalho por até cinco dias (38,2\%), devido a problemas de saúde $(41,4 \%)$. Conclusão: percebeu-se presença de processos de adoecimento entre os profissionais, os quais podem interferir na qualidade e segurança do cuidado prestado.
\end{abstract}

Descritores: Nível de Saúde; Saúde do Trabalhador; Padrões de Prática em Enfermagem.

\begin{abstract}
Objective: to analyze the sociodemographic profile, health conditions, and nurses' work characteristics. Methods: a cross-sectional study conducted with 152 nurses from a university hospital. We used a structured instrument analyzed using descriptive statistics. Results: nurses considered their health good (50.7\%), performed physical activity (53.3\%), did not smoke (92.8\%), did not consume alcohol (56.6\%), had some health problems (63.2\%) and used medication daily (54.6\%). There was a higher number of professionals working in the outpatient clinic (17.8\%) and emergency medicine (15.8\%), who worked in the studied hospital for up to five years (61.2\%), were employees subject to the Consolidation of Labor Laws (74.3\%), without another employment relationship (54.6\%), day shift (56.6\%), had a six-hour schedule (36.2\%), reported absence from work for up to five days (38.2\%), due to health problems (41.4\%). Conclusion: we noticed the presence of illness processes among professionals, which can interfere in the quality and safety of care. Descriptors: Health Status; Occupational Health; Practice Patterns, Nurses'.
\end{abstract}




\section{Introdução}

Durante a história da humanidade, o trabalho sempre emergiu como importante elemento de construção social, permitindo reflexão e reestruturação da identidade e subjetividade das pessoas ${ }^{(1)}$. Devido aos múltiplos sentidos atribuídos a essa atividade, a relevância deste transcende à esfera individual, em que atua como fonte de renda, autoestima e crescimento pessoal, para representar componente fundamental ao crescimento e desenvolvimento da sociedade ${ }^{(2)}$.

A dinâmica do processo de trabalho reflete diretamente o contexto sociocultural vivenciado por uma sociedade, adaptando-se de acordo com as transformações ocorridas em cada setor econômico, as quais interferem de forma singular sobre a oferta e demanda de produtos e/ou serviços, e acerca da inserção, absorção e redução do contingente de trabalhadores ${ }^{(3)}$. Em relação ao trabalho da enfermagem, a assistência é prestada por equipe, com diferentes níveis técnicos e hierárquicos, em que o enfermeiro, profissional de nível superior, é responsável pelas ações de gerenciamento, supervisão e realização de cuidados com maior complexidade ${ }^{(4)}$.

0 ambiente de trabalho apresenta potencial influência sobre a saúde do trabalhador e a qualidade e segurança da assistência prestada ao paciente ${ }^{(5)}$. Assim, a precarização das condições laborais na enfermagem, apesar de frequentes, sobretudo, no ambiente hospitalar, causam prejuízos na saúde e qualidade de vida do profissional, gerando intenso sofrimento, elevação dos riscos ocupacionais e aumento no número de reações adversas e acidentes de trabalho( ${ }^{(2)}$.

As próprias características do trabalho da enfermagem em hospitais podem desencadear processos de adoecimento, haja vista que a prática desse profissional é permeada pela convivência constante com o sofrimento humano e a morte, em que tais situações podem interferir de forma negativa sobre o trabalho e gerar prejuízos psicológicos para o indivíduo ${ }^{(6-7)}$.

Nesse sentido, os agravos à saúde de enfermeiros se desenvolvem de maneira multicausal, em que diversos fatores podem estar inter-relacionados e po- tencializar as experiências de sofrimento durante a prática laboral, o que requer a realização de estudos que proporcionem compreensão mais ampliada a respeito da problemática e dos determinantes envolvidos nessa interação ${ }^{(8)}$.

Assim, o estudo objetivou analisar o perfil sociodemográfico, as condições de saúde e as características do trabalho de enfermeiros.

\section{Métodos}

Trata-se de estudo transversal e quantitativo, realizado entre outubro e novembro de 2017, em hospital universitário, localizado no município de João Pessoa, Paraíba, Brasil. Este serviço de saúde se apresenta como hospital-escola, fundado em 1980, o qual possui 220 leitos, 80 consultórios e quadro de, aproximadamente, 1.100 servidores, além da capacidade de realização mensal de 20 mil atendimentos, 250 cirurgias, 50 mil exames e 700 internações.

A população deste estudo foi composta pelos enfermeiros atuantes no referido hospital. 0 cálculo da amostra foi baseado no quantitativo de profissionais com diploma de graduação em Enfermagem, registrados no Cadastro Nacional de Estabelecimentos de Saúde, do Sistema Único de Saúde, referente ao quadro de servidores da instituição, totalizando 252 enfermeiros.

O tamanho da amostra foi definido utilizando-se do cálculo para populações finitas com proporções conhecidas, tendo-se como base a margem de erro de $5 \%$ (Erro=0,05), com grau de confiabilidade de 95\% $\left(\alpha=0,05\right.$, que fornece $\left.Z_{0,05 / 2}=1,96\right)$ e proporção de participantes de $50 \%(p=0,5)$, uma vez que se utiliza esse valor como regra geral para os casos em que não se estabelece uma variável desfecho específica para análise. Desta forma, a amostra do estudo foi composta por 152 enfermeiros.

Definiram-se como critérios de inclusão: possuir vínculo empregatício ativo com o hospital e estar exercendo atividade profissional de enfermeiro no serviço há pelo menos seis meses. Definiu-se como critério de exclusão: apresentar-se em período de fé- 
rias, afastamento ou licença maternidade durante o período de coleta de dados.

Para coleta de dados, inicialmente, ocorreu o contato com os enfermeiros, no hospital, durante os períodos de intervalo dos plantões ou na entrada e saída dos profissionais, a fim prestar orientações sobre os objetivos da pesquisa, solicitar a participação no estudo e agendar o melhor horário e local para o encontro, respeitando a disponibilidade de cada um. No dia agendado, solicitou-se a assinatura do Termo de Consentimento Livre e Esclarecido e realizou-se a distribuição dos instrumentos autoaplicáveis para os enfermeiros, sendo estabelecido prazo máximo de até sete dias para devolução destes.

Aplicou-se instrumento para obtenção de dados referentes ao perfil sociodemográfico, condições de saúde e aspectos relacionados ao trabalho, cuja elaboração foi embasada no referencial da Psicodinâmica do Trabalho, que aborda os processos de saúde-doença no ambiente de trabalho ${ }^{(9)}$. Desta forma, as variáveis analisadas foram: sexo, idade, conjugalidade, renda pessoal, autopercepção da saúde, prática de atividade física, tabagismo, consumo de bebidas alcoólicas, presença de doença ou problema de saúde, uso diário de medicamentos, unidade/setor de atuação, tempo de trabalho no hospital, tipo de vínculo empregatício, presença de outro vínculo, turno, escala de trabalho, absenteísmo, período de ausência e motivo para a falta.

Os dados foram armazenados em planilha eletrônica, estruturada no Programa Microsoft Excel, com dupla digitação, visando garantir a confiabilidade na compilação dos dados. Em seguida, foram organizados, codificados, importados e processados pelo aplicativo Statistical Package for the Social Science for Windows, versão 22.0. A análise dos dados foi realizada por meio da frequência absoluta e relativa, limites inferior e superior do Intervalo de Confiança (IC) a 95\% e utilização do Teste de Qui-Quadrado para k-proporções entre as variáveis qualitativas dentro de cada grupo. 0 nível de significância utilizado para as análises estatísticas foi $5 \%(p<0,05)$.

0 estudo foi desenvolvido de acordo com o pre- conizado pela Resolução no 466/2012, do Conselho Nacional de Saúde, e suas complementariedades. A pesquisa foi aprovada pelo Comitê de Ética em Pesquisa do Hospital Universitário Lauro Wanderley, da Universidade Federal da Paraíba, conforme parecer no 2.259.018/17 e Certificado de Apresentação para Apreciação Ética no 69841417.8.0000.5183, sendo conduzido de acordo com os padrões éticos exigidos.

\section{Resultados}

Neste estudo, observou-se maior número de enfermeiros do sexo feminino $(91,4 \%)$, com idade média de 39,3 $\pm 0,96$ anos, casados ou com união estável $(62,5 \%)$ e renda pessoal entre $\mathrm{R} \$ 5.000$ e $\mathrm{R} \$ 7.999$ $(65,1 \%)$. Evidenciou-se que a maior parte dos enfermeiros investigados percebiam a própria saúde como boa $(50,7 \% ; p<0,001)$, realizavam atividade física regular (53,3\%; $p=0,417)$, não fumam $(92,8 \% ; p<0,001)$ e não ingerem bebidas alcoólicas (56,6\%; $\mathrm{p}=0,105)$, possuíam alguma doença ou problema de saúde $(63,2 \% ; p=0,001)$, principalmente as doenças do sistema osteomuscular e do tecido conjuntivo $(31,6 \%)$ e utilizavam diariamente algum tipo de medicamento $(54,6 \%)$, com destaque para os analgésicos $(32,2 \%)$ e anti-inflamatórios (28,3\%) (Tabela 1).

Tabela 1 - Condições de saúde dos enfermeiros pesquisados. João Pessoa, PB, Brasil, 2018

\begin{tabular}{|c|c|c|c|}
\hline Variáveis & n (\%) & IC $(95 \%)^{*}$ & p-valor ${ }^{\dagger}$ \\
\hline \multicolumn{4}{|c|}{ Situação de saúde } \\
\hline Excelente & $29(19,1)$ & $13,3-26,0$ & $<0,001$ \\
\hline Boa & $77(50,7)$ & $42,7-59,3$ & \\
\hline Regular & $37(24,3)$ & $16,7-31,3$ & \\
\hline Ruim & $9(5,9)$ & $2,7-10,0$ & \\
\hline \multicolumn{4}{|c|}{ Atividade física } \\
\hline Sim & $81(53,3)$ & $46,7-62,0$ & 0,417 \\
\hline Não & $71(46,7)$ & $38,0-53,3$ & \\
\hline \multicolumn{4}{|l|}{ Tabagismo } \\
\hline Não & $141(92,8)$ & $89,3-96,7$ & $<0,001$ \\
\hline Sim & $11(7,2)$ & $3,3-10,7$ & \\
\hline \multicolumn{4}{|c|}{ Consumo de bebidas alcoólicas } \\
\hline Não & $86(56,6)$ & $48,0-64,7$ & 0,105 \\
\hline Sim & $66(43,4)$ & $35,3-52,0$ & \\
\hline \multicolumn{4}{|c|}{ Doença ou problema de saúde } \\
\hline Sim & $96(63,2)$ & $54,7-71,3$ & 0,001 \\
\hline Não & $56(36,8)$ & $28,7-45,3$ & \\
\hline \multicolumn{4}{|c|}{ Uso diário de medicamentos } \\
\hline Sim & $83(54,6)$ & $45,4-62,0$ & $<0,001$ \\
\hline Não & $69(45,4)$ & $38,0-54,6$ & \\
\hline
\end{tabular}


Os setores que apresentaram o maior número de participantes foram o ambulatório $(17,8 \%)$ e a clínica médica $(15,8 \% ; \mathrm{p}<0,001)$. Observou-se que a maioria dos enfermeiros trabalhava neste hospital por um período de um a cinco anos (61,2\%; $\mathrm{p}<0,001)$, era celetista $(74,3 \%$; $\mathrm{p}<0,001)$, não possuía outro vínculo empregatício $(54,6 \% ; p=0,194)$ e desempenhava as atividades em turno diurno (56,6\%; $\mathrm{p}=0,006)$, com escala de trabalho de seis horas diárias (36,2\%; $\mathrm{p}<0,001$ ) (Tabela 2).

Tabela 2 - Aspectos relacionados ao trabalho dos enfermeiros pesquisados. João Pessoa, PB, Brasil, 2018

\begin{tabular}{|c|c|c|c|}
\hline Variáveis & n (\%) & IC $(95 \%)^{*}$ & p-valor ${ }^{\dagger}$ \\
\hline \multicolumn{4}{|l|}{ Unidade/Setor } \\
\hline Ambulatório & $27(17,8)$ & $12,0-24,7$ & $<0,001$ \\
\hline Clínica Médica & $24(15,8)$ & $10,0-22,7$ & \\
\hline Unidade de Terapia Intensiva & $16(10,5)$ & $6,0-16,0$ & \\
\hline Centro Cirúrgico & $15(9,9)$ & $4,7-14,0$ & \\
\hline Administrativo & $13(8,6)$ & $4,0-12,7$ & \\
\hline Clínica Cirúrgica & $12(7,9)$ & $3,3-13,3$ & \\
\hline Núcleo Interno de Regulação & $11(7,2)$ & $3,3-12,0$ & \\
\hline Clínica Pediátrica & $10(6,6)$ & $2,7-10,7$ & \\
\hline Clínica Obstétrica & $8(5,3)$ & $2,0-9,3$ & \\
\hline $\begin{array}{l}\text { Departamento de Doenças Infecto } \\
\text { Parasitárias }\end{array}$ & $8(5,3)$ & $2,0-9,3$ & \\
\hline Central de Material e Esterilização & $6(3,9)$ & $1,3-7,3$ & \\
\hline $\begin{array}{l}\text { Comissão de Controle de Infecção } \\
\text { Hospitalar/Vigilância em Saúde e } \\
\text { Segurança do Paciente }\end{array}$ & $2(1,3)$ & $0,0-3,3$ & \\
\hline \multicolumn{4}{|l|}{ Tempo de trabalho no hospital (anos) } \\
\hline$<1$ & $19(12,5)$ & $7,3-18,7$ & $<0,001$ \\
\hline $1-5$ & $93(61,2)$ & $54,0-70,0$ & \\
\hline $6-10$ & $7(4,6)$ & $2,0-8,7$ & \\
\hline$\geq 11$ & $30(21,7)$ & $16,7-29,1$ & \\
\hline \multicolumn{4}{|l|}{ Tipo de vínculo empregatício } \\
\hline Celetista & $113(74,3)$ & $66,7-81,3$ & $<0,001$ \\
\hline Estatutário & $39(25,7)$ & $18,7-33,3$ & \\
\hline \multicolumn{4}{|l|}{ Presença de outro vínculo empregatício } \\
\hline Não & $83(54,6)$ & $46,7-63,3$ & 0,194 \\
\hline $\operatorname{Sim}$ & $69(45,4)$ & $36,7-53,3$ & \\
\hline \multicolumn{4}{|l|}{ Turno de trabalho } \\
\hline Diurno (manhã e tarde) & $86(56,6)$ & $48,7-64,7$ & 0,006 \\
\hline Noturno & $34(22,4)$ & $16,0-30,0$ & \\
\hline Matutino & $19(12,5)$ & $6,7-17,3$ & \\
\hline Vespertino & $13(8,6)$ & $4,7-13,3$ & \\
\hline \multicolumn{4}{|l|}{ Escala de trabalho (horas) } \\
\hline 6 & $55(36,2)$ & $28,0-43,3$ & $<0,001$ \\
\hline 8 & $18(11,8)$ & $6,7-16,7$ & \\
\hline 12 & $46(30,3)$ & $23,3-38,0$ & \\
\hline Rodízio entre 6,8 ou 12 & $33(21,7)$ & $16,0-28,7$ & \\
\hline
\end{tabular}

Sobre o absenteísmo entre os profissionais investigados, a maior parte referiu ter se ausentado do trabalho $(61,8 \% ; \mathrm{p}=0,004)$ por um a cinco dias (38,2\%; $\mathrm{p}<0,001)$, durante os três meses anteriores à coleta de dados, sendo a presença de problemas de saúde $(41,4 \% ; \mathrm{p}<0,001)$ o principal motivo para falta, conforme Tabela 3.

Tabela 3 - Características do absenteísmo no trabalho dos enfermeiros participantes da pesquisa. João Pessoa, PB, Brasil, 2018

\begin{tabular}{|c|c|c|c|}
\hline Variáveis & n (\%) & IC $(95 \%)^{*}$ & p-valor ${ }^{\dagger}$ \\
\hline \multicolumn{4}{|l|}{ Falta ao trabalho } \\
\hline Sim & $94(61,8)$ & $54,0-69,3$ & 0,004 \\
\hline Não & $58(38,2)$ & $30,7-46,0$ & \\
\hline \multicolumn{4}{|c|}{ Período que se ausentou do trabalho (dias) } \\
\hline Nenhum & $58(38,2)$ & $30,7-46,0$ & $<0,001$ \\
\hline 1 e 5 & $58(38,2)$ & $30,0-46,0$ & \\
\hline 6 e 10 & $23(15,1)$ & $8,7-20,7$ & \\
\hline$\geq 11$ & $13(8,5)$ & $9,0-10,3$ & \\
\hline \multicolumn{4}{|l|}{ Motivos para falta } \\
\hline Problemas de saúde & $63(41,4)$ & $32,7-48,0$ & $<0,001$ \\
\hline Razões pessoais & $25(16,5)$ & $10,7-23,3$ & \\
\hline Afastamento & $6(3,9)$ & $1,3-7,3$ & \\
\hline Não se aplica & $58(38,2)$ & $30,7-46,0$ & \\
\hline
\end{tabular}

\section{Discussão}

Consideraram-se como limitações do estudo o preenchimento incompleto de algumas variáveis e a ausência de devolução dos instrumentos por parcela dos profissionais. Todavia, esta pesquisa contribuiu com dados que permitem aos enfermeiros conhecer as condições de saúde dos colegas de profissão e desenvolver estratégias coletivas de promoção da saúde e prevenção de agravos. Além disso, possibilita aos gestores identificar a realidade do trabalho desenvolvido no ambiente hospitalar por enfermeiros, o que favorece a criação e/ou aprimoramento dos programas de atenção à saúde do trabalhador, com enfoque na diminuição de riscos ocupacionais e investigação precoce dos processos de desgaste. 
Participaram deste estudo 152 enfermeiros, em que a maioria avaliou a própria situação de saúde como boa e apresentava hábitos de vida saudáveis, com prática de atividade física, ausência de tabagismo ou do consumo de bebidas alcoólicas. Esses achados remetem para presença de profissionais que possuem maior preocupação com a condição particular de saúde, sobretudo, com a realização de atividades de autocuidado que promovem a saúde e previnem o surgimento de doenças ou o agravamento de problemas preexistentes $^{(10)}$.

Todavia, evidenciou-se que número elevado de enfermeiros possuía alguma doença, com destaque para os problemas musculoesqueléticos. Os enfermeiros estão susceptíveis a desenvolver problemas no sistema osteomuscular, devido à manutenção de postura inadequada e/ou prolongada, durante a realização de alguns procedimentos, pela frequência de repetição de determinadas ações e por permanecerem em pé por períodos prolongados ${ }^{(11)}$. Desta forma, a rotina intensa de trabalho impossibilita o descanso adequado para promoção do relaxamento muscular, resultando em queixas frequentes de dores nos pés e nas costas, rigidez de pescoço, problemas articulares, doenças reumáticas e dificuldade de dormir ${ }^{(12-13)}$.

Em decorrência dos problemas de saúde apresentados, muitos enfermeiros fazem uso diário de medicamentos, principalmente analgésicos e anti-inflamatórios. A utilização de tais fármacos está associada às dores crônicas sofridas pelos profissionais, resultantes do desgaste físico, do elevado ritmo de trabalho e das cargas exaustivas presentes no ambiente laboral, as quais podem provocar o surgimento de quadros de ansiedade e estresse $\mathrm{e}^{(11)}$.

No presente estudo, observou-se elevado número de enfermeiros que atuava no ambulatório e na clínica médica, correspondendo, proporcionalmente, aos setores com as maiores demandas de assistência na instituição de saúde pesquisada.

0 ambulatório é um ambiente que demanda grande número de profissionais, sobretudo de enfermeiros, haja vista que atende a múltiplas especialidades clínicas e cirúrgicas, realiza curativos, pequenas cirurgias e exames, além de prestar atendimentos multiprofissionais para assistência à saúde da população ${ }^{(14)}$. A relação ambiental, também, favorece maior quantitativo de profissionais, devido à transferência dos pacientes hemodinamicamente instáveis entre os diversos setores do hospital, o que ocasiona em enfermeiros episódios de exaustão física, psíquica e afetiva com outros trabalhadores.

Em contrapartida, a clínica médica se caracteriza como ambiente com elevada complexidade, em que os pacientes apresentam alto risco de morte, exigindo a realização de procedimentos invasivos, a administração de drogas potencialmente perigosas e o monitoramento constante por parte dos profissionais, o que requer número elevado de trabalhadores, principalmente da enfermagem, para atender às legislações sobre o dimensionamento de pessoal ${ }^{(12)}$.

A maior parte dos profissionais trabalhava no hospital por período entre um e cinco anos, com prevalência do regime celetista, justificado pela recente mudança na gestão da instituição pesquisada, a qual passou a ser responsabilidade da Empresa Brasileira de Serviços Hospitalares, no ano de 2014, o que gerou a contratação inicial de 172 enfermeiros de diferentes especialidades, sendo regidos pela Consolidação das Leis do Trabalho.

Dentre os profissionais investigados, a maioria não possuía outro vínculo empregatício, todavia, merece destaque o fato de que mais de $45,0 \%$ dos enfermeiros apresentavam dupla jornada, sendo realizada na prestação de assistência direta à população. Embora a principal justificativa para o acúmulo de vínculos empregatícios seja o baixo salário recebido pela categoria profissional ${ }^{(15)}$, os enfermeiros que atuavam no hospital estudado recebiam alta remuneração, o que difere da realidade de grande parte das instituições de saúde do país.

Nesse sentido, os enfermeiros pesquisados podem ter aderido a um novo vínculo empregatício devido à flexibilidade apresentada pelo trabalho em turnos e/ou plantões, o que permite a dedicação a outras atividades produtivas e a manutenção de elevado padrão de vida ${ }^{(1,15)}$. No entanto, a sobrecarga gerada pela 
presença de múltiplos trabalhos pode representar risco à saúde do profissional e à segurança do paciente, haja vista que, em decorrência do elevado número de horas semanais de trabalho, esses indivíduos se tornam mais cansados e estão vulneráveis ao desenvolvimento de intenso desgaste físico e mental ${ }^{(16-17)}$.

Grande parte dos enfermeiros atuava em turno diurno, com escala de seis horas diárias, correspondendo à carga de trabalho de 30 e 36 horas semanais. Em hospitais, é comum a divisão do trabalho ocorrer em forma de turnos, o que permite a prestação de assistência integral e realização de cuidados contínuos, durante as 24 horas do $\mathrm{dia}^{(16)}$. Assim, a maior parte do trabalho de enfermagem é desempenhada durante o dia, uma vez que devem ser evitadas interrupções do sono e repouso do paciente à noite, o que proporciona quantitativo maior de profissionais no turno diurno ${ }^{(2)}$.

Ao investigar o absenteísmo nos enfermeiros, observou-se que elevado número de profissionais referiu ter faltado ao trabalho nos últimos três meses anteriores à coleta de dados, sendo esta ausência por período de um a cinco dias, em decorrência de problemas de saúde.

O absenteísmo representa significativo entrave para organização do processo de trabalho, pois interfere na dinâmica das atividades, provoca o comprometimento dos recursos financeiros e influencia na qualidade e segurança da assistência prestada ${ }^{(18-19)}$. Além disso, corresponde a um indicador do perfil de saúde dos profissionais, haja vista que, por ser frequentemente resultado do adoecimento do trabalhador, permite a análise da exposição às cargas de trabalho, em cada setor hospitalar, e a implementação de intervenções precoces para melhoria das condições laborais de enfermeiros ${ }^{(18,20)}$.

\section{Conclusão}

Neste estudo, o perfil dos enfermeiros pesquisados corroborou com as características nacionais da profissão, contudo, o salário apresentado divergiu do ofertado em grande parte das instituições de saúde, principalmente hospitalares. Embora os profissio- nais tenham apresentado hábitos de vida saudáveis, a maioria possuía algum problema de saúde e fazia uso diário de medicamentos. Sobre as características do trabalho, houve predomínio dos setores do ambulatório e clínica médica, atuação por no máximo cinco anos, com vínculo apenas na instituição estudada, nos períodos manhã e tarde, correspondendo a seis horas diárias, além de ausência ao trabalho por até cinco dias, em decorrência de problemas de saúde.

A partir dos resultados, podem ser identificadas relações indiretas entre o trabalho de enfermeiros no ambiente hospitalar e a presença de processos de adoecimento, os quais repercutem de maneira negativa sobre a qualidade de vida do profissional e a segurança do cuidado prestado ao paciente, haja vista que número elevado de enfermeiros se ausentou das atividades em decorrência de problemas de saúde.

\section{Agradecimentos}

À Coordenação de Aperfeiçoamento de Pessoal de Nível Superior - Código de Financiamento 001.

\section{Colaborações}

Pimenta CJL, Vicente MC, Ferreira GRS e Frazão MCLO contribuíram com concepção, projeto, análise e interpretação dos dados e redação do artigo. Costa TF e Costa KNFM colaboraram com revisão crítica relevante do conteúdo intelectual e aprovação da versão final a ser publicada.

\section{Referências}

1. Gonçalves FGA, Souza NVDO, Zeitoune RCG, Adame GFPL, Nascimento SMP. Impacts of neoliberalism on hospital nursing work. Texto Contexto Enferm. 2015; 24(3):646-53. doi: http://dx.doi. org/10.1590/0104-07072015000420014

2. Alilu L, Zamanzadeh V, Valizadeh L, Habibzadeh $\mathrm{H}$, Gillespie M. A grounded theory study of the intention of nurses to leave the profession. Rev Latino-Am Enfermagem. 2017; 25:e2894. doi: http:// dx.doi.org/10.1590/1518-8345.1638.2894 
3. Biallas B, Froböse I, Zöller M, Wilke C. Analysis of workplace health promotion and its effect on work ability and health-related quality of life in a medium-sized business. Gesundheitswesen. 2015; 77(5):357-61. doi: http://dx.doi. org/10.1055/s-0034-1372625

4. Bowden MJ, Mukherjee S, Williams LK, DeGraves S, Jackson M, McCarthy MC. Work-related stress and reward: an Australian study of multidisciplinar pediatric oncology healthcare providers. Psychooncology. 2015; 24(11):1432-8. doi: http://dx.doi.org/10.1002/pon.3810

5. Maissiat GS, Monteiro MI, Mai S, Martins MFSV, Rech CRA, Trindade LL. Work context, job satisfaction and suffering in primary health care. Rev Gaúcha Enferm. 2015; 36(2):42-9. doi: http:// dx.doi.org/10.1590/1983-1447.2015.02.51128

6. Fereidouni Z, Dehghan A, Kalyani MN. The prevalence of depression among nurses in Iran: a systematic review and meta-analysis. Int J Med Rev. 2018; 5(4):163-7. doi: http://dx.doi. org/10.29252/IJMR-050407

7. Marques DO, Pereira MS, Souza ACS, Vila VSC, Almeida CCOF, Oliveira EC. Absenteeism - illness of the nursing staff of a university hospital. Rev Bras Enferm. 2015; 68(5):876-82. doi: http:// dx.doi.org/10.1590/0034-7167.2015680516i

8. Suliman M, Aljezawi M. Nurses' work environment: indicators of satisfaction. J Nurs Manag. 2018; 26(5):1-6. doi: http://dx.doi.org/10.1111/ jonm. 12577

9. Giongo CR, Monteiro JK, Sobrosa GMR. Psicodinâmica do trabalho no Brasil: revisão sistemática da literatura. Temas Psicol. 2015; 23(4):803-14. doi: http://dx.doi.org/10.9788/TP2015.4-01

10. Yao Y, Zhao S, An Z, Wang S, Li H, Lu L, et al. The associations of work style and physical exercise with the risk of work-related musculoskeletal disorders in nurses. Int J Occup Med Environ Health. 2019; 32(1):15-24. doi: http://dx.doi. org/10.13075/ijomeh.1896.01331

11. Waters TR, Dick RB. Evidence of health risks associated with prolonged standing at work and intervention effectiveness. Rehabil Nurs. 2015; 40(3):148-65. doi: dx.doi.org/10.1002/rnj.166

12. Gasparino RC, Guirardello EB. Professional practice environment and burnout among nurses. Rev Rene. 2015; 16(1):90-6. doi: https://doi. org/10.15253/2175-6783.2015000100012
13. Guimarães ALO, Felli VEA. Notification of health problemsamongnursingworkersinuniversityhospitals. Rev Bras Enferm. 2016; 69(3):507-14. doi: dx.doi.org/10.1590/0034-7167.2016690313i

14. Dias ICCM, Torres RS, Gordon ASA, Santana EAS, Serra MAAO. Factors associated with work accidents in the nursing team. Rev Enferm UFPE on line [Internet]. 2017 [cited Feb 6, 2020]; 11(Suppl. 7):2850-5. Available from: https://periodicos. ufpe.br/revistas/revistaenfermagem/article/ view/10943/19186

15. Souza AMN, Teixeira ER. Sociodemographic profile of nursing team at the outpatient clinic of a university hospital. Rev Enferm UFPE on line [Internet]. 2015 [cited Feb 6, 2020]; 9(suppl. 3):7547-55. Available from: https://periodicos. ufpe.br/revistas/revistaenfermagem/article/ view/10493/11353

16. Silva RM, Zeitoune RCG, Beck CLC, Martino MMF, Prestes FC, Loro MM. Chronotype and work shift in nursing workers of university hospitals. Rev Bras Enferm. 2017; 70(5):1009-15. doi: http:// dx.doi.org/10.1590/0034-7167-2016-0542

17. Souza NVD, Gonçalves FGA, Pires AS, David HMS. Neoliberalist influences on nursing hospital work process and organization. Rev Bras Enferm. 2017; 70(5):961-9. doi: http://dx.doi. org/10.1590/0034-7167-2016-0092

18. Duclay E, Hardouin JB, Sébille V, Anthoine E, Moret L. Exploring the impact of staff absenteeism on patient satisfaction using routine databases in a university hospital.J Nurs Manag. 2015;23(7):83341. doi: dx.doi.org/10.1111/jonm.12219

19. Baptista PCP, Pustiglione M, Almeida MCS, Felli VEA, Garzin ACA, Melleiro MM. Nursing workers health and patient safety: the look of nurses managers. Rev Esc Enferm USP. 2015; 49(Spe 2):122-8. doi: http://dx.doi.org/10.1590/S0080623420150000800017

20. Jonoes GAL, Colville GA, Ramnarayan P, Woolfall K, Heward Y, Morrison R, et al. Psychological impact of working in paedriatric intensive care. A UK-wide prevalence study. Arch Dis Child. 2019; 1-6. doi: dx.doi.org/10.1136/archdischild-2019-317439

\section{(cc) BY}

Este é um artigo de acesso aberto distribuído sob os termos da Licença Creative Commons 